\title{
Stereo tests as a screening tool for strabismus: which is the best choice?
}

\author{
This article was published in the following Dove Press journal: \\ Clinical Ophthalmology \\ 12 November 2014 \\ Number of times this article has been viewed
}

\section{Chiara Ancona \\ Monica Stoppani \\ Veronica Odazio \\ Carlo La Spina \\ Giulia Corradetti \\ Francesco Bandello}

Department of Ophthalmology, University Vita-Salute, Scientific Institute San Raffaele, Milano, Italy
Correspondence: Chiara Ancona Department of Ophthalmology, University Vita-Salute, Scientific Institute San Raffaele, Via Olgettina, 60, 20132 Milan, Italy

Tel +3902 26432648

Fax +3902 26433643

Email chiara.ancona@hotmail.it
Purpose: To compare four stereo tests (Lang I, Lang II, Titmus, and TNO) and assess their effectiveness. The main focus of this study is to identify the most useful stereo test as a challenging tool in the screening of strabismus.

Patients and methods: A total of 143 Caucasian subjects, 74 males (52\%) and 69 females

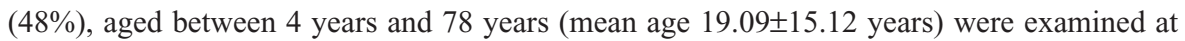
our Strabismus Service (Scientific Institute San Raffaele Hospital, Milan, Italy) and included in this observational cross-sectional study. Subjects recruited in this study were either affected by strabismus, including microstrabismic patients, or healthy volunteers. Subjects affected by ophthalmological diseases, other than strabismus, were excluded. All patients underwent both ophthalmological and orthoptic examination, including stereo tests, Hirschberg Corneal Light Reflex Test, Worth Four-Dot Test, the 4 Prism Diopter Base-Out Test, Cover Testing, Bruckner Test, visual acuity, automated refraction under $1 \%$ tropicamide cycloplegia and thereafter, posterior pole evaluation.

Results: All data were processed using the IBM SPSS Statistics, Version 2.0, to perform all statistical calculations. The main finding of this study is that Lang I stereo test achieved the highest sensitivity (89.8\%) and specificity (95.2\%) in detecting strabismus, including microstrabismus as well, compared to all the other stereoacuity tests. Furthermore, Lang I is the stereo test with the highest positive predictive value and negative predictive value, both greater than $90 \%$.

Conclusion: The stereo test with the highest sensitivity, specificity, positive predictive value, and negative predictive value is Lang I. These results suggest its applicability as a screening test for strabismus in people older than 4 years.

Keywords: stereo tests, screening, strabismus, Lang I

\section{Introduction}

Strabismus is an important medical condition with a prevalence ranging between $2 \%$ and $4 \%$ in the general population, which affects the population and causes severe complications. ${ }^{1-3}$

In most forms of strabismus, patients' motor fusion mechanisms are anomalous or are inadequate for the maintenance of ocular alignment. Therefore, eyes' inability to work together causes a reduced or absent binocular vision. ${ }^{4}$

However, in some cases in which the angle of strabismus is very small, the motor fusion amplitudes appear to be normal, whereas sensory fusion mechanisms exhibit pronounced clinical deficiencies. This form of strabismus with very small angles of deviation and relatively high degrees of binocularity has been classified as microstrabismus. ${ }^{5-9}$

The onset of strabismus when the visual system is mature can cause diplopia or confusion, while its onset when the visual system is immature can cause cortical suppression and then amblyopia. ${ }^{10,11}$ 
Stereoacuity testing is an important tool in the evaluation of vision, in order to identify the highest form of binocular coordination that can be assessed. High levels of stereoacuity are thought to influence the acquisition of sensorimotor skills in early childhood. Moreover, stereo tests measure the quality of binocularity. Since stereopsis is theoretically reduced in strabismus, this condition should be easily detected by a stereo test. $^{12-14}$

Stereoacuity tests have been used in large populationbased studies as screening tools to detect strabismus, since these tests are relatively fast, inexpensive, well accepted by both adults and children patients, and easily administrated by nonophthalmologist operators. ${ }^{15}$

However, so far there is neither general consensus nor internationally approved guidelines concerning the use of stereo tests as a screening tool for strabismus.

Nowadays, in the clinical practice a combination of different methods, as Hirschberg Corneal Light Reflex Test, Cover Testing, Worth Four-Dot Test, the 4 Prism Diopter (PD) Base-Out Test, is used to diagnose strabismus. ${ }^{4,14-17}$ The application of one of these procedures alone may be insufficient to confirm the diagnosis of the disease.

The present study was undertaken to assess a challenging method of screening for strabismus, in order to make an early diagnosis of strabismus and therefore to apply an early treatment, either medical or surgical, reaching a better prognosis.

Our experience revealed that stereo tests are a potentially useful method of screening for strabismus, considering they are easy and fast to perform and well accepted by most patients.

\section{Patients and methods}

A total of 143 Caucasian subjects, 74 males (52\%) and 69 females (48\%), aged between 4 years and 78 years (mean age $19.09 \pm 15.12$ years) were examined at our Strabismus Service (Scientific Institute San Raffaele Hospital, Milan, Italy) and included in this observational cross-sectional study. In this study, patients were consecutively admitted and the selection of the study group was done according to inclusion and exclusion criteria. Subjects recruited in this study were representative of the general population, either affected by strabismus, including microstrabismus (ten patients) and intermittent forms of strabismus (six patients), or healthy volunteers.

All patients were thoroughly informed about the purpose of the research, participation was totally voluntary, and informed consent was obtained from all participating individuals. The study protocol complied with the Declaration of Helsinki.

Subjects affected by ophthalmological diseases other than strabismus, were excluded. Patients affected by amblyopia (strabismic amblyopia or nonstrabismic amblyopia) were also excluded. Amblyopia was defined as an interocular difference in visual acuity between the two eyes $\geq 2$ lines, using Snellen charts, not attributable to any underlying structural abnormality of the eye or visual pathway.

Furthermore, patients younger than 4 years were excluded from the study. These young children could have shown a lack of collaboration that could have influenced the statistical data.

All patients underwent both ophthalmological and orthoptic examinations, including Stereo Tests, Hirschberg Corneal Light Reflex Test, Worth Four-Dot Test, the 4 PD Base-Out Test, Cover Testing, Bruckner Test, visual acuity, automated refraction under $1 \%$ tropicamide cycloplegia, and also posterior pole evaluation.

All patients were tested with all of the four stereo tests most frequently used in Europe and Italy: Lang I, Lang II, Titmus, and TNO. The tests were administrated in a randomized order, by a unique operator, who appeared to be masked to the results of both ophthalmological and orthoptic examinations. The fail-pass threshold was chosen according to the instructions of each test's manual (Lang I, Lang II, TNO fail-pass threshold) and suggestions found in literature (Titmus fail-pass threshold). ${ }^{14,16}$

Lang $I$ is a stereo test based on a combination of the random-dot technique and cylindrical gratings, consisting of a card measuring $9.5 \mathrm{~cm} \times 14.5 \mathrm{~cm}$ that contains pictures of a cat, a star, and a car, which represent disparities of 1,200, 600, and 550 seconds of arc, respectively. The test is administrated at $40 \mathrm{~cm}$ of distance; it does not require $\mathrm{red} /$ green or cross-polarized filters. Incorrect naming or no identification of one or more stereoscopic figures was considered a fail (Lang I cut-off). We consider the test negative when patients named "dog" pointing to the image of the cat. Lang II stereo test is based, as well, on the random-dot technique. It consists of a card measuring $9.5 \mathrm{~cm} \times 14.5 \mathrm{~cm}$ that contains pictures of an elephant, a car, and the moon, which represent disparities of 600, 400, and 200 seconds of arc, respectively. Similar to Lang I, the test is performed at $40 \mathrm{~cm}$ distance. This stereo test contains a control figure (a star), which is visible monocularly. The test does not require red/green or cross-polarized filters. Patients who usually wear prescription glasses have to use them during the test. Incorrect naming or no identification of one or more 
stereoscopic figures was considered a fail (Lang II cut-off). We considered the test negative when patients responded "turtle" pointing to the car or when the elephant was named as a "bear" or "cow".

In the Titmus stereo test, we considered only the second (circles) and third (animals) part, with a disparity ranging from 800 to 100 seconds of arc. The test requires crosspolarized filters (worn over prescription glasses). Failure to identify circle number 5 (100 seconds of arc) or animal A, $\mathrm{B}$, or $\mathrm{C}$ was considered a fail (Titmus cut-off).

The TNO stereo test was based on the random-dot technique, as in Lang I and II. We examined the first three plates, which represent hidden objects, easily identified by subjects with a good stereopsis. The first three plates are simple test plates with a retinal disparity of 1,900 seconds of arc. The test requires red/green filters, worn over prescription glasses. The dissociation is given by color instead of cross-polarized filters as in the Titmus test. Failure to identify just one of the hidden objects in each plate was considered a failure (TNO cut-off). We considered the test negative when patients confused rhombus with square.

A complete ocular and orthoptics evaluation was performed by a second operator, who appeared masked to the results of stereo tests. Strabismus was defined as any heterotropia either constant or intermittent. Diagnosis of strabismus was assessed by a combination of Cover Test, Hirschberg Corneal Light Reflex Test, Worth Four-Dot Test, and 4 PD Base-Out Test. ${ }^{4,14-17}$ The Cover Test is used to dissociate binocular fusion and determine the full deviation, tropia plus phoria. The Hirschberg Test, or Corneal Light Reflex Test, assesses eye alignment by noting the location of the corneal light reflex within the pupil. With normal orthotropic alignment, the light reflexes are slightly decentered nasally, but they are symmetrically located within each pupil. The Worth Four-Dot Test consists of two green lights, one red light, and one white light. The normal fusion response is seeing four lights, two red and two green. Another normal response is one red light, two green lights, and one light that flickers between green and red. The light that flickers is the white light that is seen by both eyes. Patients with acquired strabismus and diplopia will see five lights: three green and two red. Patients with cortical suppression report seeing either three green lights or two red lights, depending on which eye is fixing. Patients with large scotomas will suppress the distance Worth FourDot. Patients with the monofixation syndrome have a small central suppression scotoma and peripheral fusion. They suppress the distance Worth Four-Dot as the dots fall within the scotoma. The results of this test will tell the examiner if there is diplopia or suppression, suggesting a nondiscernible central stereoscopic vision. The association of Worth Four-Dot Test with the other two tests allowed us to identify microstrabismus also. In children younger than 10 years (54 patients), the 4 PD Base-Out Test was performed to complete the evaluation. This test consists of placing a 4 PD base-out prism over one eye. In normal subjects, the 4 base-out prism induces fusional convergence. Patients without motor fusion and large regional suppression show no movement of either eye when the prism is placed over the nondominant eye and a version movement of both eyes in the direction of the apex of the prism when the prism is placed over the fixing eye. Patients with the monofixation syndrome and a small central scotoma usually show no movement when the $4 \mathrm{PD}$ prism is placed over the nondominant eye.

Refraction measurements were conducted on all subjects included in the study after a pharmacological cycloplegia with the instillation of one drop of tropicamide $1 \%$, three times, 5 minutes apart. The refraction was evaluated 30 minutes after the last dose.

The receiver operating characteristic (ROC) analysis and the corresponding area under the curve were reported for stereoacuity tests in detecting strabismus. Specificity, sensitivity, and both positive and negative predictive values (NPVs) were calculated for each stereo test, and the corresponding $95 \%$ confidence intervals (CIs) were reported. All data were processed using the IBM SPSS Statistics, Version 2.0 (SPSS Inc., Chicago, IL, USA).

\section{Results}

All the 143 patients examined were included in this report and none were excluded, since all subjects were able to complete either the stereoacuity testing or the orthoptic and ophthalmological evaluation. In other words, all patients underwent all the examinations and tests and none of them left the study because of being incapable of finishing the tests. The mean age of patients enrolled in the study was $19.09 \pm 15.12$ years (age range 4-78 years). There were slightly more males $(74,52 \%)$ than females $(69,48 \%)$ in the sample. Among all patients included in the study, 59 (41\%) were affected by strabismus and $84(59 \%)$ were nonstrabismic healthy volunteers, tested by Hirschberg Corneal Light Reflex Test, Cover Testing, and Worth Four-Dot Test.

Ten patients were affected by microstrabismus and six patients were affected by intermittent strabismus. As already described in the previous paragraph, in some selected cases (54 patients), the 4 PD Base-Out Test was performed in order to complete the diagnosis of strabismus. 
Table I Stereo tests results showing the sensitivity, specificity, and PPVs and NPVs with $95 \% \mathrm{Cl}$

\begin{tabular}{lllll}
\hline & Lang I (95\% CI) & Lang II (95\% CI) & Titmus (95\% CI) & TNO (95\% CI) \\
\hline Sensitivity & $89.8 \%(79.2-95.6)$ & $84.7 \%(73.2-92)$ & $83.1 \%(71.3-90)$ & $79.7 \%(67.6-88.1)$ \\
Specificity & $95.2 \%(88-98.5)$ & $79.8 \%(69.9-87)$ & $83.3 \%(73.8-89.9)$ & $86.9 \%(77.9-92.7)$ \\
PPV & $93 \%(82.8-97.7)$ & $74.6 \%(63-83.6)$ & $77.8 \%(66-86.4)$ & $81 \%(69-89.2)$ \\
NPV & $93 \%(85.3-97)$ & $88.2 \%(78.8-93.9)$ & $87.5 \%(78.3-93.3)$ & $85.9 \%(76.8-91.9)$ \\
\hline
\end{tabular}

Abbreviations: $\mathrm{Cl}$, confidence interval; NPV, negative predictive value; PPV, positive predictive value.

In our study, Lang I achieved the highest sensitivity, settled at 89.8\% (95\% CI 79.2-95.6), compared with Lang II (84.7\%, 95\% CI 73.2-92), Titmus (83.1\%, 95\% CI 71.3-90), and TNO (79.7\%, 95\% CI 67.6-88.1).

Furthermore, Lang I appeared to be the most specific stereo test with a specificity of $95.2 \%$ (95\% CI 88-98.5), whereas Lang II achieved a specificity of $79.8 \%(95 \%$ CI 69.9-87). Titmus and TNO showed the least specificity, $83.3 \%$ (95\% CI 73.8-89.9) and 86.9\% (95\% CI 77.9-92.7), respectively.

The results for positive predictive value (PPV) were 93\% (95\% CI 82.8-97.7) for Lang I, 74.6\% (95\% CI 63-83.6) for Lang II, 77.8\% (95\% CI 66-86.4) for Titmus, and 81\% (95\% CI 69-89.2) for TNO.

In this study, the NPV was also calculated. Lang I was found to be the most testable ( $93 \%, 95 \%$ CI 85.3-97) compared to Lang II (88.2\%, 95\% CI 78.8-93.9), Titmus (87.5\%, 95\% CI 78.3-93.3), and TNO (85.9\%, 95\% CI 76.8-91.9).

The authors found Lang I to be the stereo test with higher sensitivity (89.8\%), specificity (95.2\%), PPV (93\%), and NPV (93\%). All these results are shown in both Table 1 and Figure 1.

The area under ROC curve analysis revealed a statistically significant superiority of Lang I test in detecting strabismus, including microstrabismus, when compared with all the other tests: 0.92 versus $0.82,0.83$, and 0.83 for
Lang I, Lang II, Titmus, and TNO, respectively $(P=0.0001)$ (Figure 2).

\section{Discussion}

Currently, there is neither general consensus nor internationally approved guidelines concerning stereo tests as a screening tool for strabismus. Poor documentation of stereo anomalies and unreported selection of participants disclose a serious problem in the clinical desire to generalize results of binocular vision research to a wider population. Previous works on the screening of strabismus using stereo tests have shown disparate results regarding their application as a screening tool and the most useful among all. ${ }^{14,17-26}$ Stereoacuity has been measured by various tests, and there are conflicting reports on their validity in the evaluation of strabismic and microstrabismic patients. There is some agreement that tests based on the random-dot technique, such as the TNO and Lang tests, are superior to the Titmus and Randot stereo tests. This belief is supported by the evidence that the stereo tests based on the random-dot technique analyze real stereopsis generated from truly binocular interaction. ${ }^{26}$

Ohlsson et a $\mathrm{a}^{16}$ studied five different stereo tests, Lang II, Frisby, Randot, Titmus, and TNO, for the screening of strabismus and amblyopia. None of these tests were suitable as screening tests because sensitivity for strabismus was lower

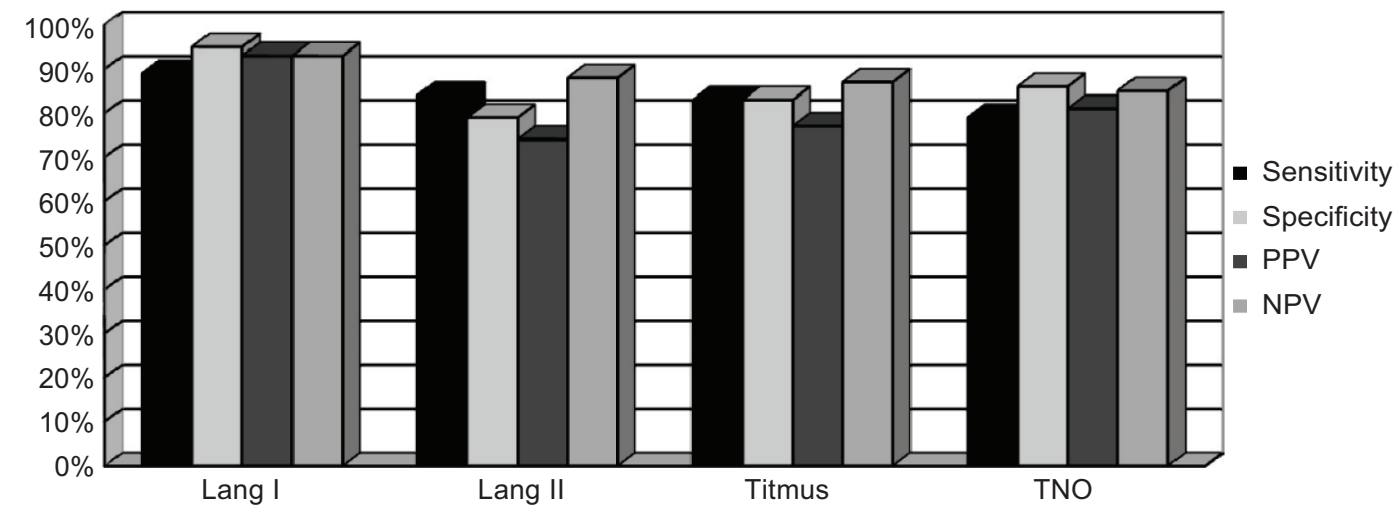

Figure I Stereo tests results showing the sensitivity, specificity, and positive and negative predictive values for strabismus expressed in percentages. Abbreviations: NPV, negative predictive value; PPV, positive predictive value. 


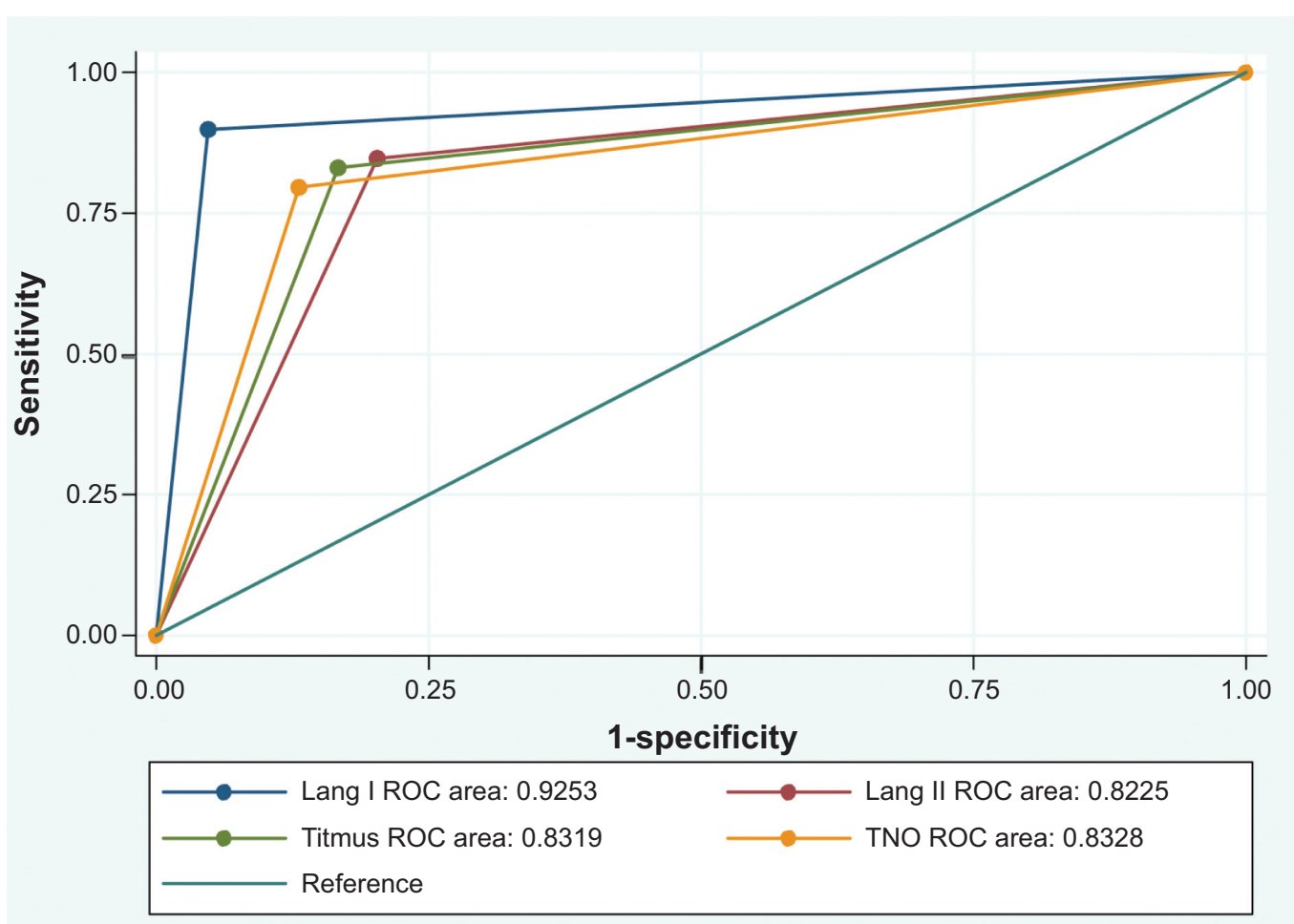

Figure 2 Stereo test receiver operating characteristic (ROC) curve and area under ROC curve.

Notes: Shows all details of the ROC analysis results. The Lang I stereo test discloses the major AUC (area under ROC curve) of 0.92 , compared to the Lang II (0.82), Titmus and TNO testing $(0.83)$. All results appeared statistically significant $(P=0.000 \mathrm{I})$.

than $80 \%$, while PPVs were lower than $40 \%$ for all the stereo tests. Strabismus passed undetected in some cases. Authors remarked that results have been affected by circumstances in the testing situation. Children were tested during school hours, leading to a lower concentration level. Furthermore, these young patients were screened with a large number of tests in the same day.

Moreover, in a further study, Ohlsson et $\mathrm{al}^{14}$ presented the effectiveness of Lang II stereo card as a screening tool for amblyopia and strabismus in children aged between 12 years and 13 years. Authors claimed that Lang II is not considered an effective method of screening for strabismus because it does not reliably distinguish between nonstrabismic patients and children affected by both strabismus and microstrabismus. Furthermore, Lang II reported a sensitivity of $82 \%$ and a PPV lower than $40 \%$. Ohlsson et al suggested that the low sensitivity could be caused by the memorization of images.

Lang II was also studied by Huynh et $\mathrm{al}^{17}$ in order to assess the accuracy of this stereo test as a screening tool for strabismus. The test achieved a sensitivity of $29 \%$ (95\% CI 26-31) in the diagnosis of strabismus. In conclusion, the authors did not recommend the sole use of Lang II stereo test in the screening of strabismus.
In literature, there are few data related to the effectiveness of Lang I stereo test in the detection of strabismus. ${ }^{18,21}$ Most of the previous papers in literature analyzed stereo tests as screening for both strabismus and amblyopia. Therefore, results regarding the effectiveness of stereo tests in the diagnosis of strabismus could appear partially masqueraded. In order to assess the relative diagnostic abilities of the four tests, we only analyzed the effectiveness of stereo tests for strabismus, excluding subjects affected by amblyopia, as already described in the previous paragraph.

In our study, we have tested the applicability of Lang I in the screening of strabismus, compared to the effectiveness of three other stereo tests, Lang II, Titmus, and TNO. We did not find any difference of compliance related to the age of the subject tested. Both strabismic and microstrabismic patients and healthy volunteers demonstrated high compliance, and none of them were excluded from the study for lack of collaboration. This point is supported by the exclusion from the study sample of children younger than 4 years, because children older than that age should possess the cognitive strategies to interpret the most difficult pictures presented to them through stereo tests. Moreover, Heron et $\mathrm{al}^{27}$ found that performance on the Randot and TNO tests was adult-like 
in 7-year-old children. Hofstetter and Bertsch ${ }^{28}$ could not $^{2}$ find any age-related differences in stereo threshold tested in 242 subjects aged between 8 years and 46 years.

Using the area under the curve of ROC curves, we determined the individual tests' capacity to detect strabismus, and then compared the four tests. Lang I stereo test showed the highest sensitivity (89.8\%), specificity (95.2\%), PPV (93\%), and NPV (93\%) compared to the other stereo tests examined (Lang II, Titmus, and TNO).

It is not easy to determine the parameters that point to why Lang I resulted superior to the others. Possible explanations could be as follows: Lang I is easier and faster to perform, it does not need red/green or cross-polarized filters, and as a random-dot test it does not present monocular clues (which, for example, are present in the contour stereoacuity tests like Titmus). The most difficult thing is to explain the different results between Lang I and Lang II: they are both random dot, they both do not need filters, and furthermore, Lang II seconds of arc (600-200) should correspond to a higher sensitivity of Lang II compared to Lang I seconds of arc (1,200-550), which can correspond to a lower sensitivity of Lang I. A hypothesis could be that when we test a patient with Lang II, he/she directs his/her attention mostly on the star visible monocularly, misinterpreting the other figures.

Our findings on the effectiveness of stereo tests in the diagnosis of strabismus differ from previously published data and in comparison to the other studies showed some advantages. First of all, we selected a homogeneous sample, including just patients affected by strabismus and excluding subjects affected by amblyopia or other forms of ocular diseases. For this reason, our results were not affected by disparities due to amblyopia and other pathologies. Patients younger than 4 years were excluded from the study. These young children could have shown a lack of collaboration conditioning the statistical data. Moreover, tests were presented to patients in a randomized order, avoiding that the order of the presentation could interfere with the analysis. In addition, the operator performing stereo tests was the same throughout the duration of this observational study. Furthermore, the operator performing stereo tests could not be influenced by the result of ocular and orthoptics evaluation, since he was masked to the results of the visits.

Finally, we underline that for the first time Lang I stereo test was compared to Lang II, Titmus, and TNO and the diagnosis of strabismus was confirmed by a combination of Hirschberg Corneal Light Reflex Test, Cover Testing, Worth Four-Dot Test, and the 4 PD Base-Out Test. No other previous studies used all these tests together in order to make strabismus diagnosis.

On the other hand, we acknowledge that our study suffers from some limitations. First of all, we mention the smaller sample size compared to the other studies and the exclusion of children younger than 4 years. These young patients were excluded because of the difficulty in interpreting their answers to stereo tests to confirm the diagnosis. The poor collaboration could affect the results, creating discrete disparities in the statistical calculations. The diagnosis of strabismus in early childhood is useful so as to perform an early treatment, either medical or surgical. Another limitation is the validity of stereo tests only for the diagnosis of constant strabismus and not for intermittent strabismus. In fact, according to our hypothesis, the rate of false negatives could be caused by the inclusion in the study group of subjects affected by intermittent strabismus (especially intermittent exotropia). Therefore, these forms of strabismus could contribute to lower the rate of sensitivity of the stereo tests studied.

In consideration of the high sensitivity, specificity, PPVs, and NPVs of Lang I and the homogeneity of the results, we believe that this stereo test could be suitable for the screening of strabismus. Such comforting results have never been reached before, in any of the previous studies. Based on these findings, we can suggest the use of Lang I stereo test in the screening of patients older than 4 years.

We understand that a good and effective screening test should include children younger than 4 years, and for this reason, it is desirable for the future to evaluate if stereo tests could be used to screen these patients. Our aim is to find a screening test for these children, not affected by poor agerelated compliance, since the early diagnosis of strabismus is important in the management of the disease.

Nevertheless, these preliminary results should be confirmed by increasing our study population and reducing the age limit as much as possible to apply stereo tests earlier, in order to obtain a more useful and applicable screening test.

\section{Conclusion}

We gave special attention to identifying a screening test for strabismus because this disease is a medical condition with a relevant prevalence in the general population $(2 \%-4 \%)$, causing severe complications when untreated, depending on the age of onset. The most disabling of these complications is amblyopia because it is irreversible if diagnosed when the plastic period of the development of the visual pathway is over. Stereoacuity studies the binocular coordination. High 
levels of stereoacuity are thought to affect the development of the sensorimotor system in early childhood.

In our study, we considered Lang I in comparison to the other stereo tests for the first time and remarked that Lang I resulted in a stereo test with the highest sensitivity (89.8\%) for strabismus, which is statistically significant compared to the other stereo tests.

Lang I demonstrated to be fast, inexpensive, affordable, acceptable by all participants, easy to perform, and could be administrated by nonophthalmologists like pediatricians and orthoptists.

For these reasons, we suggest the application of Lang I stereo test as a screening method for strabismus in patients older than 4 years.

\section{Disclosure}

Francesco Bandello is an advisory board member for Allergan, Novartis Pharmaceuticals Corporation, FarmilaThea, Bayer Schering Pharma, Pfizer, Alcon, Bausch and Lomb, Genentech, Alimera Sciences, Sanofi Aventis, Thrombogenics, Hoffmann-La Roche, and Novagali Pharma. The other authors report no conflicts of interest in this work.

\section{References}

1. McKean-Cowdin R, Cotter SA, Tarczy-Hornoch K, et al. Prevalence of amblyopia or strabismus in Asian and non-hispanic white preschool children: multi-ethnic pediatric eye disease study. Ophthalmology. 2013;120:2117-2124

2. Friedman DS, Repka MX, Katz J, et al. Prevalence of amblyopia and strabismus in white and African American children aged 6 through 71 months the Baltimore Pediatric Eye Disease Study. Ophthalmology. 2009;116:2128-2134.

3. Nucci P. Pediatric ophthalomology and strabismus in Italy. $J$ AAPOS. 2004;8:220-221.

4. Wright KW, Strube YN, editors. Pediatric Ophthalmology and Strabismus. 3rd ed. New York: Oxford University Press; 2012:217-264.

5. Lang J. Microtropia. Int Ophthalmol. 1983;6:33-36.

6. Helveston EM, von Noorden GK. Microtropia: a newly defined entity. Arch Ophthalmol. 1967;78:272-281.

7. Houston CA, Cleary M, Dutton GN, McFadzean RM. Clinical characteristics of microtropia: is microtropia a fixed phenomenon? $\mathrm{Br} J$ Ophthalmol. 1998;82:219-224.

8. Cleary M, Houston CA, McFadzean RM, Dutton GN. Recovery in microtropia: implications for aetiology and neurophysiology. $\mathrm{Br} J$ Ophthalmol. 1998;82:225-231.

Clinical Ophthalmology

\section{Publish your work in this journal}

Clinical Ophthalmology is an international, peer-reviewed journal covering all subspecialties within ophthalmology. Key topics include: Optometry; Visual science; Pharmacology and drug therapy in eye diseases; Basic Sciences; Primary and Secondary eye care; Patient Safety and Quality of Care Improvements. This journal is indexed on
9. Tomaç S, Sener EC, Sanaç AS. Clinical and sensorial characteristics of microtropia. Jpn J Ophthalmol. 2002;46:52-58.

10. Von Noorden GK, Crawford ML. The lateral geniculate nucleus in human anisometropic amblyopia. Invest Ophthalmol Vis Sci. 1983;24: 788-790.

11. Von Noorden GK, Crawford ML. The lateral geniculate nucleus in human strabismic amblyopia. Invest Ophthalmol Vis Sci. 1992;33: 2729-2732.

12. Von Noorden GK, Campos EC. Binocular Vision and space perception: stereopsis. In: Von Noorden GK, Campos EC, editors. Binocular Vision and Ocular Motility. 6th ed. St Louis: Mosby; 2002:21-25.

13. Birch E, Stager D, Wright KW, et al. The natural history of infantile esotropia during the first six months of life. Pediatric Eye Disease Investigator Group. J AAPOS. 1998;2:325-328.

14. Ohlsson J, Villarreal G, Sjöström A, Abrahamsson M, Sjöstrand J. Screening for amblyopia and strabismus with the Lang II stereo card. Acta Ophthalmol Scand. 2002;80:163-166.

15. Afsari S, Rose KA, Pai AS, et al. Diagnostic reliability and normative values of stereoacuity tests in preschool-aged children. Br JOphthalmol. 2013;97:308-313.

16. Ohlsson J, Villarreal G, Abrahamsson M, Cavazos H, Sjöström A, Sjöstrand J. Screening merits of the Lang II, Frisby, Randot, Titmus, and TNO stereo tests. J AAPOS. 2001;5:316-322.

17. Huynh SC, Ojaimi E, Robaei D, Rose K, Mitchell P. Accuracy of the Lang II stereotests in screening for binocular disorders in 6-year-old children. Am J Ophthalmol. 2005;140:1130-1132.

18. Lang J. A new stereotest. J Pediatr Ophthalmol Strabismus. 1983;20: 72-74.

19. Ehrlich MI, Reinecke RD, Simons K. Preschool vision screening for amblyopia and strabismus. Programs, methods, guidelines. Surv Ophthalmol. 1983;28:145-163.

20. Reinecke RD, Simons K. A new stereoscopic test for amblyopia screening. Am J Ophthalmol. 1974;78:714-721.

21. Manny RE, Martinez AT, Fern KD. Testing stereopsis in the preschool child: is it clinically useful? J Pediatr Ophthalmol Strabismus. 1991;28:223-231.

22. Marsh WR, Rawlings SC, Mumma JV. Evaluation of clinical stereoacuity tests. Ophthalmology. 1980;87:1265-1272.

23. Schmidt PP, Kulp MT. Detecting ocular and visual anomalies in a vision screening setting using the Lang stereotest. J Am Optom Assoc. 1994;65: $725-731$.

24. Williams S, Simpson A, Silva PA. Stereoacuity levels and vision problems in children from 7 to 11 years. Ophthalmic Physiol Opt. 1988;8: 386-389.

25. Pai AS, Rose KA, Samarawickrama C, et al. Testability of refraction, stereopsis, and other ocular measures in preschool children: the Sydney Paedriatric Eye Disease Study. J AAPOS. 2012;16:185-192.

26. Simons K. Stereoacuity norms in young children. Arch Ophthalmol. 1981;99:439-445.

27. Heron G, Dholakia S, Collins DE, McLaughlan H. Stereoscopic threshold in children and adults. Am J Optom Physiol Opt. 1985;62:505-515.

28. Hofstetter HW, Bertsch JD. Does stereopsis change with age? Am J Optom Physiol Opt. 1976;53:664-667.

PubMed Central and CAS, and is the official journal of The Society of Clinical Ophthalmology (SCO). The manuscript management system is completely online and includes a very quick and fair peer-review system, which is all easy to use. Visit http://www.dovepress.com/ testimonials.php to read real quotes from published authors. 